\title{
Influence of Perceived Racial Discrimination on Health and Behaviour of Immigrant Children in British Columbia
}

\author{
M. Anne George, ${ }^{1}$ Cherylynn Bassani, ${ }^{2}$ and Robert W. Armstrong ${ }^{3}$ \\ ${ }^{1}$ University of British Columbia, Vancouver, BC, Canada V6T $1 Z 4$ \\ ${ }^{2}$ University of the Fraser Valley, Abbotsford, BC, Canada V2S 7M8 \\ ${ }^{3}$ Aga Khan University East Africa, Nairobi, Kenya \\ Correspondence should be addressed to Cherylynn Bassani, cherylynn.bassani@ufv.ca \\ Received 16 August 2011; Accepted 21 December 2011 \\ Academic Editor: Uyen Tran
}

Copyright (c) 2012 M. Anne George et al. This is an open access article distributed under the Creative Commons Attribution License, which permits unrestricted use, distribution, and reproduction in any medium, provided the original work is properly cited.

This study examines the influence of perceived discrimination on the health and behaviour of ethnic minority immigrant children in British Columbia, Canada. Using data from the New Canadian Children and Youth Study, we examine perceived discrimination experienced by the parent, family, and cultural group in Canada to test the influence of micro-, meso-, and macrolevels of discrimination on children. Families from 6 ethnic backgrounds participated in the study. Parents' perceptions of the child's health and six behavioral scales (hyperactivity, prosocial behaviour, emotional problems, aggression, indirect aggression, and a general combined behaviour scale) were examined as outcome variables. After controlling for ethnicity and background variables, our findings suggest that perceived micro- and macrodiscrimination has the greatest influence on the health and behaviour of our immigrant child sample. Variation among ethnic groups provided the largest explanation of health and behavioural discrepancies in our study.

\section{Introduction}

Ethnic-based prejudice is an unfavorable opinion or perception of the way one's ethnocultural group is regarded by others and often leads to racial or ethnic discrimination which denotes actual practice or experience. Racial discrimination is the behaviour that excludes individuals or groups based on one's racial or ethnic group identity from engaging in opportunities, or receiving services, that would otherwise be awarded to others [1]. According to Kessler and colleagues [2], a high prevalence of racial discrimination, combined with its negative strong influence on adult's mental health makes it one of the most important factors to assess when examining wellbeing of marginalized individuals.

Ethnic based prejudice and discrimination are associated with racism: the idea that negative racial opinions and behaviours are justifiable because of group differences. Often, the concepts of racial discrimination and racism are used interchangeably. Studies that examine the influence of racism on health and general wellbeing in adult populations have progressively increased since the 1980s [3, 4]. The literature suggests that perceived racial discrimination is negatively associated with behavioural, mental, and physical health statuses $[2,5,6]$.

Both Paradies' [3] and Williams et al's. [4] meta-analyses of 138 and 52 studies, respectively, examined the influence of racism on adult health (from 1980 to 2007). Both studies concluded that ample evidence exists to support the link between racism and poor health outcomes. Paradies' examination of 138 studies covered a variety of health outcomes, including mental health, health-related behaviours, and physical health, and Williams examined both physical and mental health outcomes. Behavioural and wellbeing-focused studies also support the detrimental influence of perceived racism. Kessler et al. [2] found that discrimination negatively influences the emotional wellbeing of adults belonging to marginalized ethnic groups. In addition, racial discrimination has been linked to heightened levels of depression $[2,7]$ and increased rates of morbidity and mortality [5]. 
Research is limited with respect to children and perceived discrimination. A few studies report that younger children do perceive discrimination [8-10]. Coker et al. [10] found an association between perceived ethnic or racial discrimination and increased likelihood to have symptoms of four mental health conditions (depression, attention deficit hyperactivity disorder, oppositional defiant disorder, and conduct disorder) amongst children in the fifth grade in the USA. Gee and Walsemann [6] observed that among young adults, employment discrimination is not only associated with health limitations, but also temporally ordered so that the discrimination precedes the health limitations.

Most studies examine the direct influence of racial discrimination on adult health; however, few studies illustrate that the effects of such discrimination are mediated through variables such as social status or through minor health issues that can lead to more significant health problems. This line of research has found racial discrimination to be associated with increased stress which can potentially lead to low selfesteem $[2,11]$ and increased blood pressure [12], which in turn leads to a decline in one's general mental and physical health status [13].

The majority of the literature finds a link between discrimination and poor health; however, the extant research is nevertheless limited in a number of ways. To begin, we could not find studies on the effects of discrimination on the health of immigrants and in particular children of immigrant families or of children in general, except in the area of mental health issues such as depression as noted above [8-10]. Secondly, most of this adult-based literature, and all of the child-based literature, focuses on American samples and relatively few studies from other nations can be found. In addition, the bulk of this American literature focuses on the experiences of large minority groups, such as women, those living in poverty, and racial discrimination among African American and American Indian populations.

Our research addresses the limitations currently found in the literature as we examine the influence of racial discrimination on the health and behaviour of children whose families immigrated to Canada. That is to say, not only do we focus on immigrant children, but also our research uses Canadian data, an understudied population.

\section{Theory}

Theory explaining the relationship between racial discrimination and wellbeing falls into three main models: the poor health model, the resiliency model, and the contextualized model. The poor health model posited by Williams et al. [14] suggests a negative relationship between racial discrimination and health. They suggest that poor health is a product of three paths: racism creates social status that negatively influences health; racism determines exposure to risks; racism negatively influences the individual's ability to function. The social status argument has been further developed by Ren et al. [13] who found a strong negative association between health and racial and class discrimination, health implications of discrimination due to low SES, thereby noting a complex relationship between perceived discrimination and social class.

The resiliency model has also been supported in the literature. Among this research, personal characteristics are seen to help reduce the influence, or increase the coping mechanisms, related to discrimination. For example, Dion et al. [11] found that in Toronto's Chinese community "hardiness" (composite of perceived personal control and selfesteem) buffered the effects of discrimination. Hardiness was increased by attributes such as higher education, an occupation with higher socioeconomic status, and proficiency in the English language, all of which led to a stronger sense of selfcontrol which facilitated coping.

In the contextualized model, as Karlsen and Nazroo [15] maintain, the influence that racism has on health is highly contextualized and depends not only on the type of racism experienced (individual or institutional), but also on the severity of the racist experience(s). In addition, similar to the poor health model, Karlsen and Nazroo [15] point out the need to consider the complex relationship between SES (and its derivatives) and health. As Adler and Ostove [16] discuss, while a direct positive relationship between SES and health has been observed, there are "multiple pathways" in which SES influences health. While we live in a democratic (pseudo-) egalitarian society, race and ethnicity still play a pivotal role in creating one's SES and therefore influence their overall wellbeing.

In our research, we follow the contextualized model. Drawing on Karlsen and Nazroo's [15] theoretical premises, we examine micro- (parent experiences), meso- (family experiences), and macro- (cultural group's experience) levels of discrimination. While we affirm that severity of discrimination is necessary to be examined, we are only able to measure the frequency of various forms of discrimination and racism that is not physical. Lastly, we maintain that social status is an important issue when contextualizing racism and its influence on health and wellbeing. SES may be formed differently for Canadian immigrants, as education, occupation, and income may not be as interconnected as they are for nonimmigrant Canadians. Given this issue with SES, we are interested to see the individual effects that the SES control variables (income, education, years in Canada) have on health and wellbeing.

\section{Methods}

3.1. Data. The data used in this paper are drawn from the New Canadian Children and Youth Study (NCCYS), a national longitudinal study on the health and wellbeing of immigrant children in Canada. The NCCYS includes four urban centre study sites: Montreal, Toronto, the Prairies (Winnipeg, Calgary, and Edmonton), and Vancouver (see $[17,18]$ for a description of the sampling frame). In the full study, immigrants from 16 backgrounds participated across the 4 sites. In this paper we use a subset of the full study, including those who live in and around the city of Vancouver, and includes families from The Philippines, Hong Kong, Mainland China, Afghanistan, Iran, and the Punjab region 
of India. Using the data for the Vancouver site reduces the geographical effects of where a family settles in Canada, since variables such as influences of provincial social and health services may contribute to outcome measures. Families were eligible to participate in the NCCYS if they had children in one of two age cohorts (4-6 years and 11-13 years). Children could be born either in Canada or in their country of origin; however, the family had to have immigrated to Canada within the previous 10 -year period.

Data were collected through bilingual interviewers in the home of the family. Structured interview instruments were completed by the primary caregiver (usually the mother) and by the children in the older cohort. Data collection took approximately 1-2 hours, depending on the number of eligible children in the household. Data were collected on household demographics, child health and behaviour, schooling, and pre- and postimmigrant experiences. Many questions were drawn from the National Longitudinal Survey of Children and Youth (NLSCY) [19].

NCCYS data are based on quota snowball sampling since reliable lists of immigrant children are not available. Quota sampling was also used to ensure an equal sample size from each ethnic community and for each age group of children. One hundred and eighty children were recruited from each ethnic community: 90 in the younger group (4-6 years old) and 90 in the older age cohort (11-13 years old). Families with more than one child in the target age group were included in the study.

3.2. Sample. The NCCYS sample used for these analyses is restricted to those children residing in the Vancouver area. As indicated in Table 1, the sample size for the Vancouver data is 1081; with approximately 180 children (90 from each cohort) from each of the six ethnic groups. Data were not weighted, since the use of the weight variable substantially decreased the number of respondents in the Afghani, Iranian, and Punjabi groups, and overestimated the sample in the remaining ethnic groups. Since we maintain that immigrants are not a homogenous group, and need to be viewed as heterogeneous, our analyses seek to test ethnic-based health and behavioural differences.

3.3. Measures. Descriptive results for all dependent and independent variables are listed in Table 1.

3.3.1. Dependent Variables. Seven dependent variables are assessed. The child's health was measured by a single question, with the primary caregiver rating health on a 5-point Likert-type scale. Responses were dummy coded, as either "excellent" or "not excellent," that is, any other response other than "excellent." Self-rated (or the proxy rating by parents) health scores are widely used as global measures of health and have been found to be highly predictable of mortality and the need for health care across communities and age groups, social asses, and educational backgrounds [20-24].31.4\% of the children in the sample was reported to have excellent health.
Children's behaviours were assessed via six behaviour scales: one composite behavioural scale and five subscaleshyperactivity, prosocial behaviour, emotional problems, physical aggression and indirect aggression. Scales were created and tested by NLSCY researchers and are routinely used by child researchers.

The hyperactivity/inattention scale (this scale is referred to as the hyperactivity/inattention scale by the NCCYS; herein, we refer to it as the hyperactivity/inattention scale) combines eight items. As shown in Table 1, this scale ranges from 0 (no hyperactive behaviour) to 16 (constant hyperactive behaviour) and has a mean of 2.77 and an alpha of 0.77 . The second scale, measuring prosocial behaviour, combines ten items and ranges from 0 (no prosocial behaviour) to 20 (constant prosocial behaviour) with a mean of 13.15 and an alpha of 0.84 . The prosocial scale is reverse coded from the other behavioural scales: a high score indicates more prosocial behaviour. The emotional problems scale ranges from 0 to 16 and was compiled of eight items. A higher score indicates higher level of emotional problems. This scale has a mean of 1.84 and an alpha of 0.74 . Two aggression scales were created: physical aggression/conduct disorder and indirect aggression. The physical aggression/conduct disorder scale (this scale is referred to as the physical aggression/conduct disorder scale by the NCCYS; herein we refer to this scale as the physical aggression scale) is a compilation of six items, such as "gets into many fights", is cruel or bullies" and "physically attacks people." The mean is 0.87 , range from 0 to 12 and the alpha is 0.63 . The second aggression scale, indirect aggression, combines five items related to the reactions when the child is mad at someone, such as "tries to get others to dislike that person" and "says bad things behind the other's back." The mean for the indirect aggression scale is 1.21 , range 0 to 10 and the alpha is 0.74 . In both scales a higher score indicates an increase in aggressive behaviour. For each of the five subscales, missing values (prosocial scale missing $n=50$; hyperactivity/inattention scale missing $n=25$; emotional problem scale missing $n=26$; physical aggression scale missing $n=21$; indirect aggression scale missing $n=34$ ) were imputed to the individual scale's mean.

A general behaviour scale was created by combining the five behaviour scales discussed above. Responses from each individual scale were recoded to a standard scoring system across all five scales, with a range from 0 (no behaviour problems) to 3 (behaviour problems). The prosocial scale was reverse recoded. The sub scales were then merged to create a scale that ranged from 0 to 15 , mean of 1.63 and an alpha of 0.52 . A higher score denotes more behaviour problems. A higher score denotes more behavior problems. The six scales were created and tested by NLSCY Statistics Canada analysts; however, we conducted factor analysis for each scale to determine dimensionality. Two factors were found in each of the hyperactivity, emotional problems, physical aggression, and general behaviour scales, while only one factor was found in each of the remaining scales (prosocial and indirect aggression). We maintained the original NLSCY scales, though note that they were created for and tested on general child/youth populations. 
TABLE 1: Descriptive statistics, NCCYS Vancouver site data, $n=1083$.

\begin{tabular}{|c|c|c|c|c|c|}
\hline \multicolumn{6}{|l|}{ Dependent variables } \\
\hline Continuous & Mean & SD & Items & Range & Alpha \\
\hline \multicolumn{6}{|l|}{ Behaviour scales $^{1}$} \\
\hline Hyperactive (Model 2) & 2.77 & 2.66 & 8 & $0-16$ & 0.77 \\
\hline Prosocial (Model 3) & 13.15 & 4.07 & 10 & $0-20$ & 0.84 \\
\hline Emotional problems (Model 4) & 1.84 & 2.14 & 8 & $0-16$ & 0.74 \\
\hline Physical aggression (Model 5) & 0.87 & 1.26 & 6 & $0-12$ & 0.63 \\
\hline Indirect aggression (Model 6) & 1.21 & 1.57 & 5 & $0-10$ & 0.74 \\
\hline Combined behaviour (Model 7) & 1.63 & 1.57 & 5 & $0-15$ & 0.52 \\
\hline Categorical & \multicolumn{3}{|c|}{ Percent } & \multicolumn{2}{|c|}{$N$} \\
\hline \multicolumn{6}{|l|}{ Health (Model 1) } \\
\hline Excellent & & 31.4 & & \multicolumn{2}{|c|}{340} \\
\hline Other & & 68.3 & & \multicolumn{2}{|c|}{740} \\
\hline Missing & & 0.3 & & \multicolumn{2}{|c|}{3} \\
\hline Total & & 100.0 & & \multicolumn{2}{|c|}{1083} \\
\hline \multicolumn{6}{|l|}{ Independent variables } \\
\hline Continuous & Mean & $\mathrm{SD}$ & Items & Range & Alpha \\
\hline \multicolumn{6}{|l|}{ Discrimination scales $^{1}$} \\
\hline Parental discrimination & 1.17 & 1.47 & 3 & $0-9$ & 0.76 \\
\hline Family discrimination & 0.45 & 0.83 & 6 & $0-6$ & 0.52 \\
\hline \multirow[t]{2}{*}{ Cultural discrimination } & 6.08 & 1.56 & 6 & $0-18$ & 0.80 \\
\hline & Mean & SD & Range & $N$ & Missing \\
\hline Income $^{1}$ & 5.55 & 2.99 & $0-12$ & 1083 & - \\
\hline Education & 2.54 & & $0-6$ & 1040 & 43 \\
\hline Categorical & & \multicolumn{2}{|l|}{ Percent } & \multicolumn{2}{|c|}{$N$} \\
\hline \multicolumn{6}{|l|}{ Ethnicity } \\
\hline Afghani & & 16.7 & & & \\
\hline Hong Kong Chinese & & 16.6 & & & \\
\hline Iranian & & 16.7 & & & \\
\hline Filipino & & 16.6 & & & \\
\hline Punjabi & & 16.6 & & & \\
\hline Mainland Chinese* & & 16.7 & & & \\
\hline Total & & 100.0 & & & \\
\hline Years in Canada & & & & & \\
\hline 2 years or less & & 35.0 & & & \\
\hline Over 2 years & & 65.0 & & & \\
\hline Total & & 100.0 & & & \\
\hline Gender & & & & & \\
\hline Female & & 47.8 & & & \\
\hline Male & & 52.2 & & & \\
\hline Missing & & 0.3 & & & \\
\hline Total & & 100.0 & & & \\
\hline Age & & & & & \\
\hline Younger (4-6) & & 50.1 & & & \\
\hline Older (11-13) & & 49.9 & & & \\
\hline Total & & 100.0 & & & \\
\hline
\end{tabular}


3.3.2. Independent Variables. The independent variables used in this study include three discrimination scales, ethnicity, and five background variables.

Discrimination. Following Karlsen's and Nazroo's [15] contextualized ideology of racism, we assessed the influence of three different levels of ethnic discrimination that were perceived by the parent, the family, and the ethnic group in Canada. Alphas were computed for each of the three scales, as was factor analysis. The first discrimination scale, which combined three variables measured perceived discrimination toward the parent over the last year. Parents were asked: "how often they felt stress because of the following experiences or feelings? Others discriminate against me, I am treated as an alien by other Canadians, and I am constantly reminded by others of my minority status." Response categories included: "never, sometimes, often, very often, or not applicable." The not applicable category was coded as a system missing response and the three variables were merged to create the parent discrimination scale which ranged from 0 (no discrimination) to 9 (frequent discrimination). This scale has a mean of 1.17 and an alpha of 0.76 . One factor was found among these three variables. The family discrimination scale is comprised of six variables, which asked: "in the past 12 months, has anyone in your family experienced any of the following: being unfairly or denied promotion from a job? not hired for a job for unfair reasons? being treated unfairly by the police? being unfairly discouraged from continuing education by a teacher or advisor? being treated unfairly in selling/buying a house or renting an apartment? and being treated unfairly by neighbours?" The parent responded either positively or negatively to each question. The family discrimination scale ranges from 0 (no discrimination) to 6 (highly discriminated) and has a mean of 0.45 and an alpha of 0.52 . Three factors were found among these six variables, suggesting that three latent concepts underlie these questions; however, theoretically all six of the questions are bound by experiences of being treated unfairly, or being discriminated against. As a result, we maintain that this scale has high face validity. The cultural prejudice scale measured the amount of perceived prejudice that the family's culture faces in Canada. Although cultural discrimination may be perceived, and therefore more likely to be prejudice than actual exclusion, we are using the term cultural discrimination. Six variables were used to form this scale. Parents were asked how strongly they agreed with the following statements: "Canadians tend to look down on us; People from home country portrayed in media less fairly than other groups; Landlords would rather rent an apartment to another group; People from home country treated less fairly at government offices; People from home country treated less fairly at stores; People from home country treated less fairly when applying for jobs." Respondents had the option of choosing: strongly disagree, disagree, not sure, agree, or strongly agree. Response category "not sure" was recoded to the missing response since it suggests that the respondent does not know the answer to the question. This scale ranges from 0 (no perceived discrimination) to 18 (perceived discrimination) and has a mean of 6.08, and an alpha of 0.80 . One factor was found among these six items.

Ethnicity. Ethnicity of the parent was also examined. Five dummy-coded variables measured ethnicity. Ethnicity of the respondent was measured by the country of origin that they emigrated from. Respondents and/or their children came from six countries, representing the six ethnic groups analyzed in this study: Afghanistan (16.7\%), Hong Kong Chinese (16.6\%), Punjabi (16.6\%), Iranian (16.7\%), Filipino, (16.6\%), and Mainland Chinese (16.7\%). Mainland Chinese respondents were used as the reference group since historically Mainland Chinese immigrants are British Columbia's and Canada's largest ethnic minority immigrant culture. As a result, we tend to know more about individuals in this group since more research has been conducted on this immigrant group compared to other ethnic minority groups in British Columbia or Canada.

Background Variables. Five background variables were considered. The two core components of SES, income and education, were included as continuous variables in the study. Income was measured through a closed-ended 12-item response variable that ranged from $\$ 0.00$ to over $\$ 80,000$ per year in household income. This variable is ordinal, though as income category increased the interval between income categories became larger. The average income was 5.55 , representing a household income in between $\$ 20,000$ and $\$ 29,999$ per year. The modal income was 5 , representing an income in between $\$ 20,000$ and $\$ 24,999$ per year. Missing cases $(n=28)$ were coded to the mean.

The education measure was based on the primary caregiver's (typically the mother) level of education upon entry into Canada (a high school diploma or less, a college diploma or certificate, a bachelor's degree, a master's degree, a professional degree, or a doctorate degree). The mean education was 2.5 (somewhere between a college diploma and a bachelor's degree), with $17.3 \%$ of having a college diploma/certificate, and $35.7 \%$ having a bachelor's degree. The modal education was a bachelor's degree; however, nearly $29 \%$ of primary caregiver parents had a high school diploma or lower education.

Years in Canada were also included as a background variable, since the literature supports the notion of the healthy immigrant effect $[25,26]$. That is to say, immigrants are healthiest when they first arrive to Canada and then gravitate downwards towards the national health mean over time. A cut-off period is used because it is generally understood that new-comers require a certain time period to become accustomed to their new country including finding resources such as health care, friends, and so on. Children who had be been in Canada for two years or less were dummy coded and compared to children who had been in Canada for over two years. Approximately, two-fifths of children had been in Canada for 2 years or less.

Gender and age were also included as background variables. Females (1) were dummy coded in reference to boys ( 0$)$, while older children (11-13 years old) were used as 
TABLE 2: Effect of ethnic discrimination on health and behaviour: Models 1 through 8, NCCYS 1 Vancouver site data, $n=1083$.

\begin{tabular}{|c|c|c|c|c|c|c|c|c|c|c|c|c|c|c|}
\hline & \multicolumn{2}{|c|}{$\begin{array}{c}\text { Health } \\
\text { (Model 1) }\end{array}$} & \multicolumn{2}{|c|}{$\begin{array}{c}\text { Hyperactive } \\
\text { scale } \\
\text { (Model 2) }\end{array}$} & \multicolumn{2}{|c|}{$\begin{array}{l}\text { Prosocial } \\
\text { scale } \\
\text { (Model 3) }\end{array}$} & \multicolumn{2}{|c|}{$\begin{array}{c}\text { Emotional } \\
\text { problems } \\
\text { scale } \\
\text { (Model 4) }\end{array}$} & \multicolumn{2}{|c|}{$\begin{array}{c}\text { Physical } \\
\text { aggression } \\
\text { scale } \\
\text { (Model 5) }\end{array}$} & \multicolumn{2}{|c|}{$\begin{array}{c}\text { Indirect } \\
\text { aggression } \\
\text { scale } \\
\text { (Model 6) }\end{array}$} & \multicolumn{2}{|c|}{$\begin{array}{c}\text { General } \\
\text { behaviour } \\
\text { scale } \\
\text { (Model 7) }\end{array}$} \\
\hline & $\begin{array}{l}\text { Exp } \\
(B)\end{array}$ & Wald & $b$ & $t$ & $b$ & $t$ & $b$ & $t$ & $b$ & $t$ & $b$ & $t$ & $b$ & $t$ \\
\hline Constant & -1.34 & 3.07 & 1.81 & 2.58 & 8.98 & 8.75 & 1.87 & 3.25 & 0.00 & 0.01 & 0.69 & 1.61 & 1.80 & 4.49 \\
\hline Parent & 0.98 & 0.08 & 0.09 & 1.45 & 0.18 & 2.16 & 0.08 & 1.57 & 0.05 & 1.70 & 0.10 & 2.72 & 0.10 & 2.85 \\
\hline Family & 0.87 & 1.47 & 0.04 & .34 & -0.15 & -1.01 & 0.29 & 3.42 & 0.03 & 0.67 & 0.03 & 0.54 & 0.40 & 0.66 \\
\hline Culture & 0.90 & 3.52 & 0.16 & 3.04 & 0.25 & 0.33 & 0.13 & 0.31 & 0.05 & 2.03 & 0.02 & 0.89 & 0.60 & 1.91 \\
\hline Afghani & 1.04 & 0.01 & -1.02 & -3.42 & -1.28 & -2.96 & -0.73 & -3.02 & 0.75 & 5.11 & 0.81 & 4.47 & -0.30 & -1.77 \\
\hline $\begin{array}{l}\text { Hong } \\
\text { Kong }\end{array}$ & 1.08 & 0.05 & 0.90 & 3.15 & 0.65 & 1.59 & 0.49 & 2.17 & 0.39 & 2.81 & 0.43 & 2.54 & 0.65 & 4.10 \\
\hline Iranian & 36.35 & 122.87 & -1.67 & -6.02 & 4.04 & 10.01 & -1.71 & -7.57 & 0.06 & 0.46 & -0.57 & -3.40 & -0.02 & -0.12 \\
\hline Filipino & 4.39 & 26.92 & 0.48 & 1.80 & 0.87 & 2.22 & -0.92 & -4.20 & 0.46 & 3.47 & -0.33 & -2.00 & 0.19 & 1.21 \\
\hline Punjabi & 3.45 & 17.66 & 0.62 & 2.23 & 2.17 & 5.32 & -0.49 & -2.15 & 0.60 & 4.30 & 0.27 & 1.57 & 0.55 & 3.45 \\
\hline $\begin{array}{l}\text { Less } 2 \\
\text { Years }\end{array}$ & 0.96 & 0.05 & 0.36 & 1.99 & 0.27 & 1.04 & 0.03 & 0.19 & -0.00 & -0.03 & 0.11 & 1.02 & 0.17 & 1.67 \\
\hline $\begin{array}{l}\text { Educ- } \\
\text { ation }\end{array}$ & 1.01 & 0.05 & -0.12 & -1.55 & 0.54 & 4.99 & 0.00 & 0.05 & -0.02 & -0.63 & -0.11 & -2.45 & 0.05 & 1.08 \\
\hline Income & 1.08 & 6.15 & -0.06 & -2.10 & 0.10 & 0.24 & -0.20 & -0.84 & 0.01 & 0.32 & -0.01 & -0.20 & -0.02 & -1.05 \\
\hline Female & 1.29 & 2.41 & -0.61 & -3.91 & 0.81 & 3.52 & -0.06 & -0.47 & -0.29 & -3.75 & 0.08 & 0.80 & 0.02 & 0.24 \\
\hline Older & 1.26 & 1.79 & -0.82 & -4.78 & 0.49 & 1.95 & 0.06 & 0.43 & -0.40 & -4.64 & -0.24 & -2.25 & -0.15 & 0.23 \\
\hline$\overline{R^{2}}$ & \multicolumn{2}{|c|}{0.26} & \multicolumn{2}{|c|}{0.15} & \multicolumn{2}{|c|}{0.23} & \multicolumn{2}{|c|}{0.11} & \multicolumn{2}{|c|}{0.08} & \multicolumn{2}{|c|}{0.10} & \multicolumn{2}{|c|}{0.14} \\
\hline
\end{tabular}

Model 1 reports Cox and Snell $R^{2}$ and significance of chi squared to test the significance of the model. Models 2 through 7 report adjusted $R^{2} .{ }^{1}$ Mainland China ethnic origin is the reference group. $P \leq 0.05(t \leq 1.96)$. Bold coefficients denotes a statistical significance of 0.05 or greater. Model 1: dependent variable $=$ health $(1$ : excellent health $)$. Model 2: dependent variable $=$ hyperactivity scale $(0-16)$. Model 3: dependent variable = prosocial behaviour scale $(0-$ 20). Model 4: dependent variable $=$ emotional problems scale (0-16). Model 5: dependent variable $=$ physical aggression scale (0-12). Model 6: dependent variable $=$ indirect aggression scale $(0-10)$. Model 7 : dependent variable $=$ general behaviour scale $(0-15)$.

the reference group (1) in their comparison to younger children (4-6 years old) (0).

3.4. Statistical Analyses. Seven models were tested in this paper. Both logistic and regular ordinary least squares (OLS) regression analyses were conducted. Model 1, the analysis that examined excellent health, used logistic regression to test the likelihood of a child having excellent health. Models 2 through 7 used OLS regression to assess the influence of discrimination, controlling for ethnicity, and other background variables across all of the behaviour scales. Unstandardized beta coefficients and the corresponding $t$-values are reported in Table 2. For the sake of readability, bolded betas represent coefficients with $t$-values that were statistically significant.

Analyses were completed using SPSS 18. Alpha was set at $0.05(t, 1.96)$.

This study was approved by research ethics review committees at the Universities of Alberta, British Columbia, Calgary, Manitoba, Toronto, and Winnipeg and at McGill University.

\section{Results}

Results of the seven regression analyses are illustrated in Table 2 .
4.1. Model Fit. As indicated in Table 2, Models 1 and 3 have a moderately sized, statistically significant explained variance. Twenty-six percent $(26 \%)$ of the variance surrounding excellent health and $23 \%$ of the variance surrounding prosocial behaviour was explained by the variables included in the models. In the remaining models, a smaller amount of variance was explained, though all $R^{2}$ coefficients are statistically significant. Fifteen percent $(15 \%)$ of the variation surrounding hyperactivity and $14 \%$ of the variation surrounding the general behaviour scale was explained. In Models 4 (measuring emotional problems), 5 (physical aggression) and 6 (indirect aggression), 11\%, 8\%, and 10\%, respectively, of variance was explained.

4.2. Ethnic Discrimination. Three measures of ethnic discrimination were assessed, that is, discrimination against the parent, the family and the culture that the parent belonged to. As illustrated in Table 2, discrimination does not have a consistent statistically significant influence on the various health measures. It appears that the individual and cultural level measures of discrimination have the most influence on children's health and behaviour.

Discrimination experienced by the parent had an effect in three of the seven models. This form of discrimination was associated with an increase on the indirect aggression $(b=0.10)$ and general behaviour scales $(b=0.10)$, meaning 
that racial discrimination at the individual level worked to increase the child's indirect aggression and his/her general behaviour score (recall a higher score on the behaviour scale equates with more behavioural issues). Interestingly, the effect of parent's perceived discrimination on the child's prosocial behaviour score worked to increase the child's score. This unexpected finding accounted for only a small amount of variance.

Ethnic discrimination that family members experienced was significant in one of the seven models, as it worked to increase the level of the child's emotional problems level. For every 1-point increase on the family discrimination scale, the child's emotional problems score increased by 0.29 points.

In four of the models perceived discrimination against the respondent's culture worked to negatively influence health and behaviour. For every 1-point increase on the cultural discrimination scale, children experienced a 0.16 point increase on the hyperactivity scale, a 0.05 point increase on the direct aggression scale, and a 0.60 point increase on the general behaviour scale. Children that were exposed to cultural discrimination were also less likely to report having excellent health. For every 1-point increase on the cultural discrimination scale, these children were 0.90 times as likely to report having excellent health.

4.3. Ethnicity. Ethnicity had sizable, statistically significant effects on children's health and behaviour, meaning that children's health and behaviour vary based on the ethnic group that they belong to. Five trends can be noted in Table 2 when assessing the ethnicity of the child.

First, children in families who emigrated from Hong Kong tended to have higher behaviour scores than children from families from Mainland China. Children from Hong Kong had higher scores on the hyperactivity ( 0.90 points), emotional problems ( 0.49 points), and physical ( 0.39 points) and indirect ( 0.43 points) aggression scales, in addition to higher general behavioural scores ( 0.65 points) when contrasted to children with a Mainland Chinese background. No difference was found between these two groups when prosocial behaviour or health was examined. While these statistically significant differences exist, it is important to note that average behavioural scores are quite small for the entire sample, which suggests that parents in our sample perceived their children as having few behavioural issues.

The second trend is the reported higher health scores among the Iranian children. Iranian children displayed significantly higher levels of health in five of the seven models. The Iranian children were 36.35 times more likely than children from Mainland China to report excellent health. In addition, they were more likely to have lower hyperactivity $(-1.67)$, emotional problems $(-1.71)$, and indirect aggression scores $(-0.57)$. In addition, Iranian children had a higher level of prosocial behaviour. On average, they scored 4.04 points higher on the prosocial scale when contrasted to youth with a Mainland Chinese background, holding all other variables in the equation constant. When assessing physical aggression and general behaviour, Iranian youths did not score differently than the Mainland Chinese youth.
The third trend found in our results is that children with Iranian, Filipino, and Punjabi backgrounds reported to have better health than the Mainland Chinese comparison group. As stated, Iranian children were 36 times more likely to report having excellent health, while Filipino and Punjabi children were four and three times more likely, respectively, to report having excellent health when compared to the Mainland Chinese group. Children with Afghani and Hong Kong Chinese backgrounds were no more likely than the comparison group to report having excellent health.

Next, Table 2 also illustrates that all children, with the exception of those from Hong Kong, had less emotional problems than the Mainland Chinese group.

The fifth trend focuses on physical aggression. With the exception of children with Iranian backgrounds (who were no different from children from Mainland China), all children displayed higher physical aggression scores than children with Mainland Chinese backgrounds. Physical aggression scores were $0.75,0.39,0.46$, and 0.60 points higher among children with Afghani, Hong Kong Chinese, Filipino, and Punjabi backgrounds, when contrasted to the comparison group.

4.4. Background Variables. Five background variables were considered in this study: length of time living in Canada (two years or less versus living in Canada for more than two years up to ten years), household income, primary caregiver's education upon arriving in Canada, gender of the child, and age group of the child.

Children who lived in Canada for two or fewer years were not found to differ in their health or behaviour when compared to children who had lived in Canada for two to ten years. The education of the primary caregiver parent was found to positively influence behaviour scores in Models 2, 3, and 6. As education level increased by 1-point, hyperactivity and indirect aggression scores declined by 0.12 and 0.11 points, respectively. In addition, prosocial behaviour scores increased by 0.54 points as education increased by 1 point. Household income also worked to reduce hyperactivity scores: as income increased by one level, hyperactivity declined by 0.06 points. Additionally, as income increased children were $8 \%$ more likely to report having excellent health. Sex and age of the child had widespread statistically significant effects on health and behaviour compared to the other background variables. Females had lower hyperactivity $(b=-0.61)$ and physical aggression $(b=-0.29)$ scores than boys did, and higher prosocial behaviour scores $(b=0.81)$. They also were $29 \%$ more likely to be in excellent health compared to boys. Lastly, older children were found to have lower hyperactivity $(b=-0.82)$, physical aggression $(b=$ $-0.40)$, and indirect aggression scores $(b=-0.24)$ than were younger children. They were also found to have higher prosocial (0.49) scores.

\section{Discussion}

In examining the direct influences of micro-, meso- and macrolevels of discrimination, findings were inconsistent 
although it appears that individual or cultural discrimination is more influential on children's health than is family discrimination. Our measures of discrimination relied on parent perceived discrimination. We suggest that future research examines the child's perspective.

Interestingly, we found that parents' perceived discrimination slightly increased the child's prosocial behavior scores. Similar to what Dion et al. [11] argue in their resiliency model of discrimination, this relationship might be explained if the parent was intentionally buffering, or over compensating, their child from discrimination that they had themselves experienced. Future research will need to explore this finding.

Our findings are concordant with those reported elsewhere [17] in illustrating considerable differences between the various ethnic communities with respect to health outcomes. Future research may provide a better understanding of whether such findings represent real differences between cultural groups or are an artifact of parents' reporting on their child's health and behaviours. For example, Afghani children (who are all refugees) had lower scores on the emotional problems scale than the Mainland Chinese comparison group. This finding may be attributed to the resiliency that many refugee children exhibit in their host country; alternatively, Afghani parents might portray their children as enduring fewer emotional problems in Canada, compared to parents from other ethnic groups. Another example of this in the results was that parents from Hong Kong reported more child-behaviour issues than parents from other immigrant populations. This may be an artifact of parents' reporting, with parents from Hong Kong possibly having higher expectations of their children. This is a limitation of the current study, that is, we lack an understanding of whether differences in parent's reporting is based on cultural differences or subjectivity. Given that the questions were standard, asked in the parent's original language, and objective gives us some confidence that real cultural differences may exist. Future work is needed in this area.

Another limitation of the study is that the severity of discrimination is not recorded. Future research will need to focus on this aspect of discrimination.

The strength of this study is that it contributes to an understudied area, that is, the influence of perceived discrimination amongst immigrant children's health in British Columbia. The NCCYS is the first study to focus entirely on the health and wellbeing of immigrant and refugee children in Canada. Much of the literature on discrimination comes from researchers in the USA, who focus on perceived racism amongst African Americans, a population which is large, well-established, and has a different history from immigrants and refugees, who by definition are newly settled in their new country. In addition, research on perceived discrimination in the area of child health is limited.

Canada, along with other western nations, is a receiving country with respect to immigrant populations. Understanding the wellbeing of all its children, including those of immigrant families, is important to not only the wellbeing of these families but also the wellbeing of the nation.
Furthermore, as with the research that examines adults, discrimination experienced by young adults and children is also associated with poor mental health. While we have examined the influence of ethnic discrimination experienced by the particular ethnic group, the family, and the primary care giver parent, future research should focus on discrimination faced by the youth. While culture, family, and parents all have an important influence on youth health and behavior, it is important to understand the influence that discrimination experienced by the child has on their health and behaviour.

This paper has noted that significant child health and behavioural differences exist between the various immigrant ethnic groups that we have examined. These results demonstrate the importance in understanding differences between immigrant children, rather than considering them as a homogeneous entity. This is particularly noteworthy given that the current literature tends to group immigrants together and not examine ingroup differences. Such differences are vital not only in the development of theoretical and analytic models, but also more importantly in preparing for and treating ingroup differences.

As shown by Gee and Walsemann [6], discrimination precedes negative health outcomes, and as such it is essential that we understand the effects of discrimination amongst children so that we may ultimately attempt to prevent discrimination, but also the consequences of such discrimination.

\section{Acknowledgments}

This paper is a product of the New Canadian Children and Youth Study (Principal Investigators: Morton Beiser, Robert Armstrong, Linda Ogilvie, Jacqueline Oxman-Martinez and Joanna Anneke Rummens), a national longitudinal survey of the health and wellbeing of more than 4,000 newcomer immigrant and refugee children living in Montreal, Toronto, Winnipeg, Edmonton, Calgary, and Vancouver. The NCCYS is a joint collaboration between university researchers affiliated with Canada's four Metropolis Centres of Excellence for research on immigration and settlement, and community organizations representing Afghani, Hong Kong Chinese, Mainland Chinese, Latin American (El Salvadorean, Guatemalan, Colombian), Ethiopian, Haitian, Iranian, Kurdish, Lebanese, Filipino, Punjabi, Serbian, Somali, Jamaican, Sri Lankan Tamil, and Vietnamese newcomers in Canada. Major funding for the project has been provided by the Canadian Institutes for Health Research (CIHR Grants FRN43927 and PRG-80146), Canadian Heritage, Citizenship and Immigration Canada (CIC), Health Canada, Justice Canada, Alberta Heritage Foundation for Medical Research, Alberta Learning, BC Ministry of Social Development and Economic Security, BC Ministry of Multiculturalism and Immigration, Conseil Quebecois de la Recherche Sociale, Manitoba Labour and Immigration, and the Montreal, Prairies, and Ontario Metropolis Centres of Excellence for research on immigration and settlement. 


\section{References}

[1] R. T. Schaefer, Racial and Ethnic Groups, Harper and Collins Publishers, 1993.

[2] R. C. Kessler, K. D. Mickelson, and D. R. Williams, "The prevalence, distribution, and mental health correlates of perceived discrimination in the United States," Journal of Health and Social Behavior, vol. 40, no. 3, pp. 208-230, 1999.

[3] Y. Paradies, "A systematic review of empirical research on selfreported racism and health," International Journal of Epidemiology, vol. 35, no. 4, pp. 888-901, 2006.

[4] D. R. Williams, H. W. Neighbors, and T. S. Jackson, "Racial/ ethnic discrimination and health: findings from community studies," American Journal of Public Health, vol. 98, supplement, pp. 1541-1549, 2008.

[5] R. Clark, N. B. Anderson, V. R. Clark, and D. R. Williams, "Racism as a stressor for African Americans: a biopsychosocial model," American Psychologist, vol. 54, no. 10, pp. 805-816, 1999.

[6] G. Gee and K. Walsemann, "Does health predict the reporting of racial discrimination or do reports of discrimination predict health? Findings from the National Longitudinal Study of Youth," Social Science \& Medicine, vol. 68, no. 9, pp. 16761684, 2009.

[7] B. K. Finch, B. Kolody, and W. A. Vega, "Perceived discrimination and depression among Mexican-origin adults in California," Journal of Health and Social Behavior, vol. 41, no. 3, pp. 295-313, 2000.

[8] G. H. Brody, Y. F. Chen, V. M. Murry et al., "Perceived discrimination and the adjustment of African American youths: a fiveyear longitudinal analysis with contextual moderation effects," Child Development, vol. 77, no. 5, pp. 1170-1189, 2006.

[9] R. L. Simons, V. Murry, V. McLoyd, K. H. Lin, C. Cutrona, and R. D. Conger, "Discrimination, crime, ethnic identity, and parenting as correlates of depressive symptoms among African American children: a multilevel analysis," Development and Psychopathology, vol. 14, no. 2, pp. 371-393, 2002.

[10] T. R. Coker, M. N. Elliott, D. E. Kanouse et al., "Perceived racial/ethnic discrimination among fifth-grade students and its association with mental health," American Journal of Public Health, vol. 99, no. 5, pp. 878-884, 2009.

[11] K. L. Dion, K. K. Dion, and A. W.-P. Pak, "Personality-based hardiness as a buffer for discrimination-related stress in members of Toronto's Chinese community," Canadian Journal of Behavioural Science, vol. 24, no. 4, pp. 517-536, 1992.

[12] N. Krieger and S. Sidney, "Racial discrimination and blood pressure: the CARDIA study of young black and white adults," American Journal of Public Health, vol. 86, no. 10, pp. 13701378, 1996.

[13] X. S. Ren, B. C. Amick, and D. R. Williams, "Racial/ethnic disparities in health: the interplay between discrimination and socioeconomic status," Ethnicity and Disease, vol. 9, no. 2, pp. 151-165, 1999.

[14] D. R. Williams, R. Lavizzo-Mourey, and R. C. Warren, "The concept of race and health status in America," Public Health Reports, vol. 109, no. 1, pp. 26-41, 1994.

[15] S. Karlsen and J. Y. Nazroo, "Relation between racial discrimination, social class, and health among ethnic minority groups," American Journal of Public Health, vol. 92, no. 4, pp. 624-631, 2002.

[16] N. E. Adler and J. M. Ostove, "Socioeconomic status and health: what we know and what we don't," in Socioeconomic Status and Health in Industrial Nations: Social, Psychological and Biological Pathways, N. E. Adler, M. Marmot, B. S. McEwen, and J. Stewart, Eds., vol. 896, pp. 3-15, Annals of the New York Academy of Science, 1999.

[17] M. Beiser, H. Hamilton, J. A. Rummens et al., "Predictors of emotional problems and physical aggression among children of Hong Kong Chinese, Mainland Chinese and Filipino immigrants to Canada," Social Psychiatry and Psychiatric Epidemiology, vol. 45, no. 10, pp. 1011-1021, 2010.

[18] M. Beiser, N. Zilber, L. Simich et al., "Regional effects on the mental health of immigrant children: results from the New Canadian Children and Youth Study (NCCYS)," Health \& Place, vol. 17, no. 3, pp. 822-829, 2011.

[19] Statistics Canada and Ministry of Industry, National Longitudinal Survey of Children and Youth: Growing up in Canada, Ottawa, Canada, 1996.

[20] A. Quesnel-Vallée, "Self-rated health: caught in the crossfire of the quest for "true" health?" International Journal of Epidemiology, vol. 36, no. 6, pp. 1161-1164, 2007.

[21] J. B. Dowd and A. Zajacova, "Does the predictive power of selfrated health for subsequent mortality risk vary by socioeconomic status in the US?" International Journal of Epidemiology, vol. 36, no. 6, pp. 1214-1221, 2007.

[22] M. Huisman, F. Van lenthe, and J. Mackenbach, "The predictive ability of self-assessed health for mortality in different educational groups," International Journal of Epidemiology, vol. 36, no. 6, pp. 1207-1213, 2007.

[23] A. Singh-Manoux, A. Dugravot, M. J. Shipley et al., "The association between self-rated health and mortality in different socioeconomic groups in the GAZEL cohort study," International Journal of Epidemiology, vol. 36, no. 6, pp. 12221228, 2007.

[24] E. L. Idler and Y. Benyamini, "Self-rated health and mortality: a review of twenty-seven community studies," Journal of Health and Social Behavior, vol. 38, no. 1, pp. 21-37, 1997.

[25] R. Dinovitzer, J. Hagan, and P. Parker, "Choice and circumstance: social capital and planful competence in the attainments of immigrant youth," Canadian Journal of Sociology, vol. 28, no. 4, pp. 463-488, 2003.

[26] X. Ma, "The first ten years in Canada: a multi-level assessment of behavioural and emotional problems of immigrant children," Canadian Public Policy, vol. 28, no. 3, pp. 395-418, 2002. 


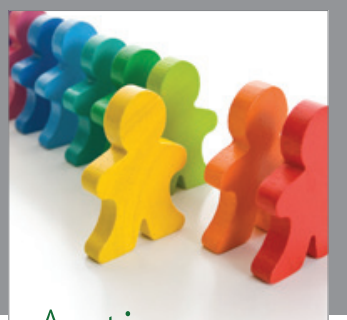

Autism

Research and Treatment
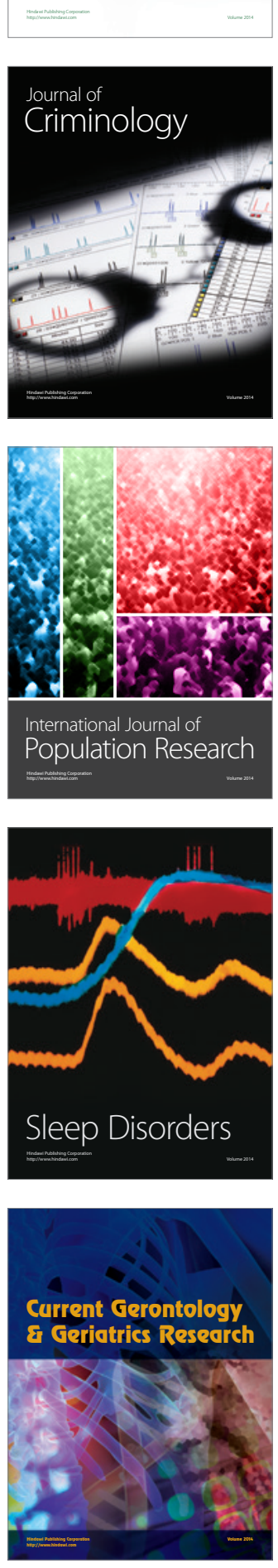
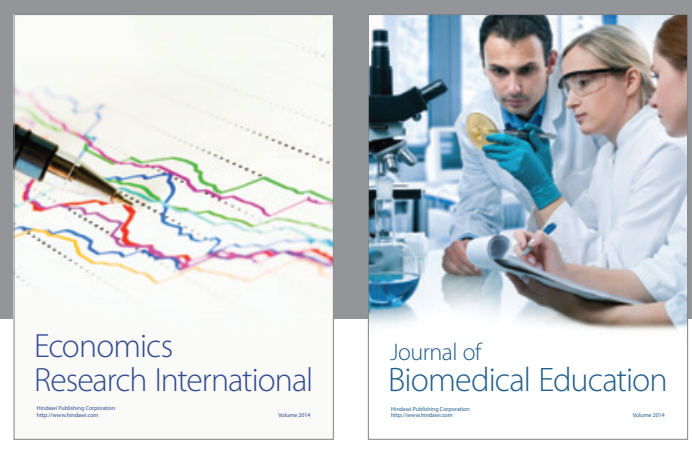

Journal of

Biomedical Education

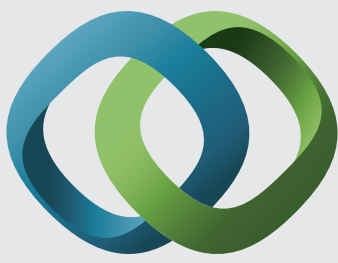

\section{Hindawi}

Submit your manuscripts at

http://www.hindawi.com
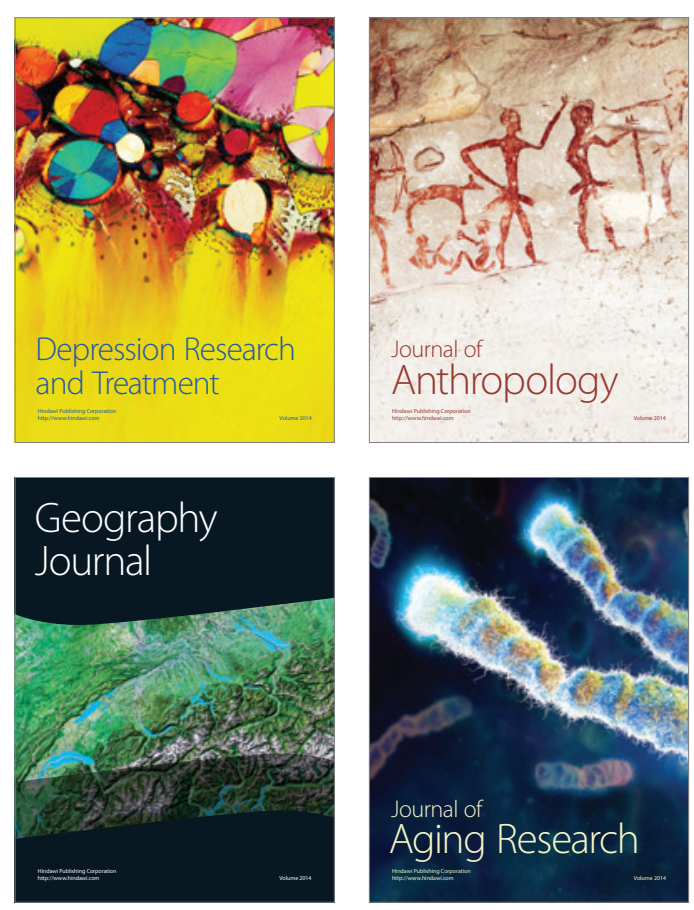

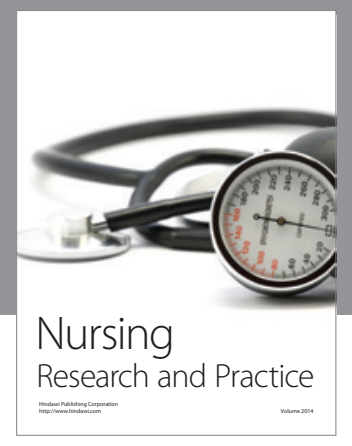

Nursing

Research and Practice

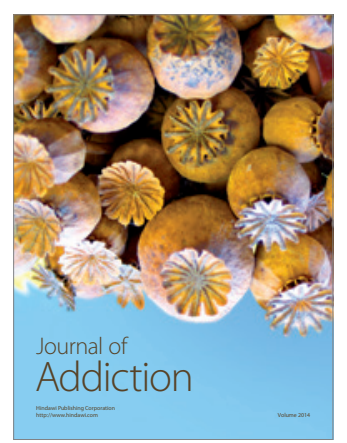

Child Development

Research

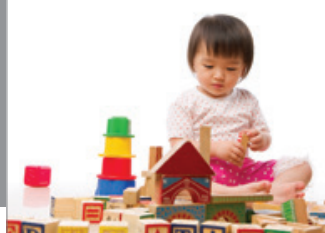

迥
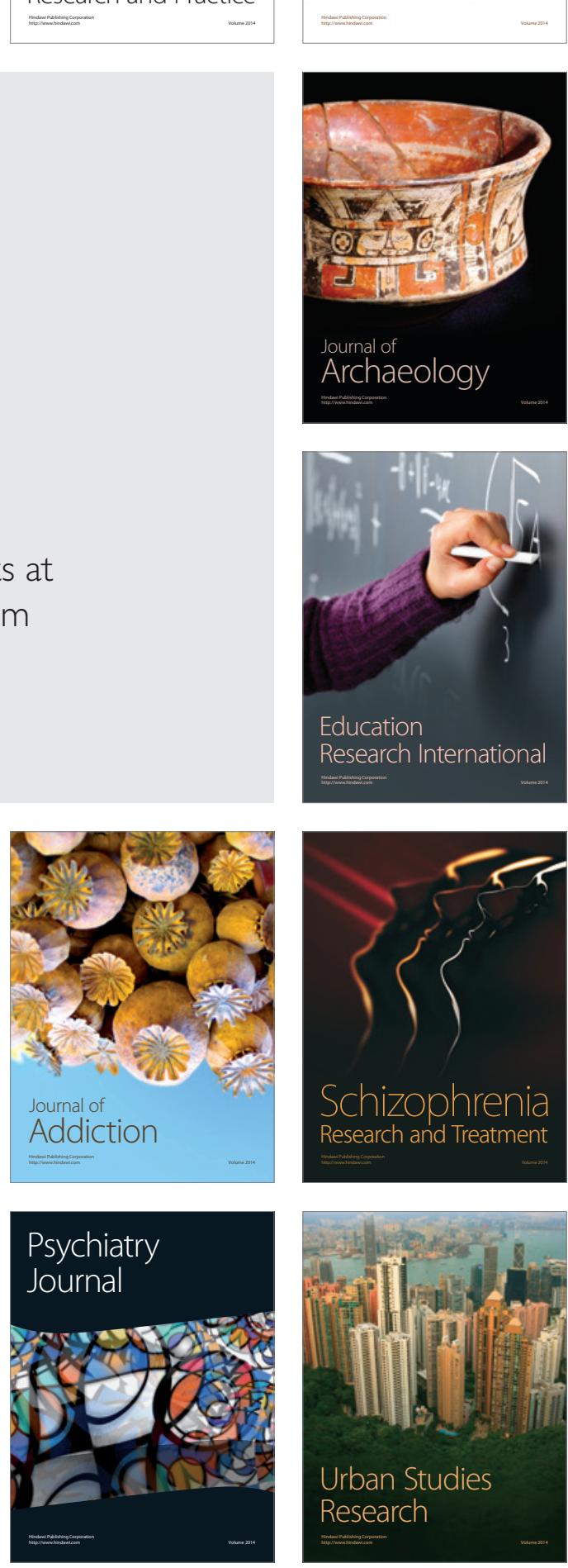